\title{
The anticancer properties of iron core-gold shell nanoparticles in colorectal cancer cells
}

This article was published in the following Dove Press journal:

International Journal of Nanomedicine

30 August 2013

Number of times this article has been viewed

\author{
Ya-Na Wu' \\ Ping-Ching $\mathrm{Wu}^{\prime}$ \\ Li-Xing Yang' \\ Kyle R Ratinac ${ }^{2}$ \\ Pall Thordarson ${ }^{4}$ \\ Kristina A Jahn ${ }^{2}$ \\ Dong-Hwang Chen ${ }^{3}$ \\ Dar-Bin Shieh ${ }^{1,5,6}$ \\ Filip Braet ${ }^{2,7}$ \\ 'Institute of Oral Medicine and \\ Department of Stomatology, \\ National Cheng Kung University \\ Hospital, College of Medicine, \\ Tainan, Taiwan; ${ }^{2}$ Australian Centre \\ for Microscopy and Microanalysis, \\ University of Sydney, Sydney, NSW, \\ Australia; ${ }^{3}$ Department of Chemical \\ Engineering, National Cheng Kung \\ University, Tainan, Taiwan; ${ }^{4}$ School \\ of Chemistry, University of New \\ South Wales, Sydney, NSW, Australia; \\ ${ }^{5}$ Center for Micro/Nano Science and \\ Technology, National Cheng Kung \\ University, Tainan, Taiwan; ${ }^{6}$ Advanced \\ Optoelectronic Technology Center, \\ National Cheng Kung University, \\ Tainan, Taiwan; ${ }^{7}$ School of Medical \\ Sciences (Discipline of Anatomy \\ and Histology), The Bosch Institute, \\ University of Sydney, Sydney, NSW, \\ Australia
}

Correspondence: Ya-Na Wu Institute of Oral Medicine and Department of Stomatology, National Cheng Kung University Hospital, College of Medicine, Tainan 70I, Taiwan

Tel +886 62353535 , ext 5410

Fax +88662359885

Email yana.wu@gmail.com

\begin{abstract}
Previously, iron core-gold shell nanoparticles (Fe@Au) have been shown to possess cancer-preferential cytotoxicity in oral and colorectal cancer (CRC) cells. However, CRC cell lines are less sensitive to $\mathrm{Fe} @$ Au treatment when compared with oral cancer cell lines. In this research, $\mathrm{Fe} @ \mathrm{Au}$ are found to decrease the cell viability of CRC cell lines, including Caco-2, HT-29, and SW480, through growth inhibition rather than the induction of cell death. The cytotoxicity induced by Fe@Au in CRC cells uses different subcellular pathways to the mitochondria-mediated autophagy found in Fe@Au-treated oral cancer cells, OECM1. Interestingly, the Caco-2 cell line shows a similar response to OECM1 cells and is thus more sensitive to Fe@Au treatment than the other CRC cell lines studied. We have investigated the underlying cell resistance mechanisms of Fe@Au-treated CRC cells. The resistance of CRC cells to Fe@Au does not result from the total amount of Fe@ $\mathrm{Au}$ internalized. Instead, the different amounts of $\mathrm{Fe}$ and $\mathrm{Au}$ internalized appear to determine the different response to treatment with Fe-only nanoparticles in Fe@Au-resistant CRC cells compared with the Fe@Au-sensitive OECM1 cells. The only moderately cytotoxic effect of Fe@Au nanoparticles on CRC cells, when compared to the highly sensitive OECM1 cells, appears to arise from the CRC cells' relative insensitivity to $\mathrm{Fe}$, as is demonstrated by our Fe-only treatments. This is a surprising outcome, given that Fe has thus far been considered to be the "active" component of Fe@Au nanoparticles. Instead, we have found that the Au coatings, previously considered only as a passivating coating to protect the Fe cores from oxidation, significantly enhance the cytotoxicity of Fe@Au in certain CRC cells. Therefore, we conclude that both the Fe and Au in these core-shell nanoparticles are essential for the anticancer properties observed in CRC cells.
\end{abstract}

Keywords: cancer therapy, Fe, gold-coated iron, nanoparticles, differential cytotoxicity

\section{Introduction}

With the development of nanomedicine, magnetic nanomaterials have great potential for use as a combined therapeutic and diagnostic tool in medical applications, especially in cancer treatment. ${ }^{1-3}$ In diagnostic applications, magnetic nanomaterials can be detected by magnetic resonance imaging (MRI) ${ }^{4,5}$ and X-ray computed tomography (CT). ${ }^{5}$ In therapeutic applications, metallic nanomaterials can not only serve as drug carriers, ${ }^{6}$ but they can also be applied as thermotherapeutic probes under radial high-frequency fields. ${ }^{7}$ Among those current magnetic nanomaterials, iron oxidebased nanoparticles have a key advantage with high biocompatibility. ${ }^{3,8,9}$ Indeed, iron oxide-based nanoparticles used as MRI contrast agents were among the first approved clinical nanomaterials. ${ }^{10}$

Nevertheless, elemental iron has a much larger magnetic susceptibility than iron oxides; therefore, it has immense potential if it is able to be stabilized in a nanoscale form. As a result, Carpenter et al designed iron nanoparticles passivated by a gold 
coating in order to stabilize the iron core and delay its oxidation. ${ }^{11,12}$ These iron core-gold shell nanoparticles (Fe@, $\mathrm{Au}$ ) have been proposed for use as MRI contrast agents, ${ }^{13}$ CT contrast agents, ${ }^{14}$ molecular diagnostic tools, ${ }^{15}$ magnetic guidable drug-carriers, ${ }^{14}$ and thermotherapeutic probes. ${ }^{16}$ Intriguingly, our previous research demonstrated that $\mathrm{Fe} @ \mathrm{Au}$ showed cancer-preferential cytotoxicity toward oral cancer in vitro and in vivo, which was caused by the nonoxidized iron in the nanoparticles. ${ }^{17}$ Furthermore, we determined that the nanoparticles caused their cytotoxic effect in OECM1 through mitochondria-mediated autophagy. ${ }^{18}$ Similarly, Thurber et al ${ }^{19}$ have demonstrated that Fe doping of $\mathrm{ZnO}$ nanoparticles improved their ability to selectively kill leukemia cells. They suggested that pure iron possesses potential as a cancerselective anticancer agent, in accordance with our results.

Although Fe@Au nanoparticles show a similar cancerpreferential cytotoxicity in colorectal cancers (CRC), it seems that $\mathrm{CRC}$ is much more resistant to Fe@Au treatment, compared with oral cancer cells. ${ }^{17}$ However, the underlying mechanism of CRC resistance to the Fe@Au treatment is still unclear. Unraveling the puzzle of differential responses of different cancer types to Fe@Au treatment should lead to more efficient anticancer strategies in the future. Therefore, the research presented here aims to investigate the differential response of CRC to $\mathrm{Fe@Au} \mathrm{treatment.} \mathrm{We} \mathrm{first} \mathrm{investigated}$ the underlying molecular mechanisms of the cytotoxicity induced by Fe@Au in CRC and then explored the role of different elements in anticancer properties that we observed.

\section{Materials and methods Synthesis of Fe@Au and Fe nanoparticles}

The core-shell structures were synthesized and characterized by using a sequential synthesis technique provided by reverse micelles in a cetyltrimethylammonium bromide (CTAB), n-butanol and water system. ${ }^{17,18,20-23}$ The Fe nanoparticles were synthesized by two microemulsion solutions ([water]:[detergent] =2.54). Briefly, the size of the particles was controlled by the molar ratio of the water to surfactant $\left(\omega=\left[\mathrm{H}_{2} \mathrm{O}\right] /[\mathrm{CTAB}]\right)$. The reaction process was carried out under flowing argon gas. In the first step, two microemulsion solutions were prepared using 0.2 $\mathrm{M} \mathrm{FeSO}_{4}$ (aqueous [aq]) (catalog number F8633, Sigma-Aldrich, St Louis, MO, USA) and 0.5 $\mathrm{M} \mathrm{NaBH}_{4}$ (aq) (catalog number 480886, Sigma-Aldrich), respectively, in a ratio of $\omega=2.54$. These solutions were mixed together using a magnetic stirrer at a speed of $260 \mathrm{rpm}$, and the solution turned black upon the reduction of the iron. In the second step, the shell of gold was synthesized. Two additional micellar solutions were prepared using 0.05 $\mathrm{M} \mathrm{HAuCl}_{4}$ (aq) (catalog number 254169, SigmaAldrich) and $0.8 \mathrm{M} \mathrm{NaBH}_{4}(\mathrm{aq})$, respectively, with $\omega=3.8$. These solutions were purged by argon to minimize the oxygen contamination caused during oxidation of the iron core. These solutions were added to the reaction mixture from Step 1. In this case, the size of micelles in Step 2 solutions was increased slightly $(\omega=3.24)$, within the reaction mixture to allow for the growth of gold shell on the core. The reaction was stirred under flowing argon for 2 hours. The nanoparticles were ultrasonically washed in argon-purged ethanol three times and then magnetically collected. The particles were then dried under vacuum and sealed in argon. The Fe nanoparticles were synthesized in a similar way; however, only two microemulsion solutions with $0.05 \mathrm{M}$ of $\mathrm{FeSO}_{4}$

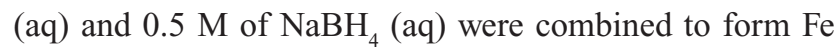
nanoparticles, as described in Step 1. ${ }^{24,25}$ The Fe nanoparticles were then washed and recovered in the argon-purged absolute ethanol solutions for the following experiments.

\section{Cell lines}

The OECM1 cell line, established from a male Taiwanese oral cancer patient with a betel nut chewing history, ${ }^{26}$ was cultured in Roswell Park Memorial Institute medium (RPMI) 1640 (catalog number 72400047, Invitrogen, Carlsbad, CA, USA), supplemented with 10\% fetal bovine serum (FBS) and antibiotic-antimycotic (AA) reagent (catalog number 15240-062, Invitrogen). The human colorectal cancer cell lines Caco-2, HT-29, and SW480 were obtained from American Type Culture Collection (ATCC; Middlesex, UK). Caco-2 and HT-29 cells were kept in Advanced Dulbecco's Modified Eagle's Medium (catalog number 12491023, Invitrogen), supplemented with 10\% FBS, 2 mM of L-glutamine (catalog number 35050-079, Invitrogen), and 1×AA. The SW480 cells were grown in L-15 (Leibovitz) medium (catalog number 11415-064, Invitrogen), supplemented with 10\% FBS and AA. For the media-substitution experiments, Caco-2, HT-29, and SW480 were first cultured in a mixture of original culture medium and RPMI 1640 in a ratio of 3:1 for 2 days, followed by $1: 1$ for 2 days, and 1:3 for another 2 days. Then, the cells were cultured in pure RPMI 1640 medium.

\section{WST-I cytotoxicity assay}

To evaluate the cytotoxicity of Fe@Au nanoparticles, cells growing in the log phase were seeded at a density of 5,000 cells per well in a 96-well culture plate. Prior to each in vitro experiment, particles were resuspended in phosphate-buffered saline (PBS) at $10 \mathrm{mg} / \mathrm{mL}$ as the stock solution. The 3-methyladenine (3-MA) (catalog number M9281, Sigma-Aldrich) was prepared 
at a concentration of $200 \mathrm{mM}$, as $20 \times$ stock solutions in PBS, and stored at $-80^{\circ} \mathrm{C}$. The 3-MA was dissolved by heating prior to usage, at a final concentration of $10 \mathrm{mM}$ cyclosporin A (CsA) (catalog number C3662, Sigma-Aldrich) was prepared at a concentration of $5 \mathrm{mM}$ as $1,000 \times$ stock solutions in dimethyl sulfoxide (DMSO); it was then stored at $-20^{\circ} \mathrm{C}$. Cells were cultured and treated with different concentrations of nanoparticles or an assigned cotreatment. In our previous study, the best time for assessing cell viability was 48 hours after the treatment; ${ }^{17,18}$ therefore, we tested for cell viability at the 48 hour time point throughout this experiment. The cell viability tests were performed by the WST-1 (water-soluble tetrazolium salt) cytotoxic assay, according to the user manual (catalog number 630118, Clontech Laboratories, Madison, WI, USA). ${ }^{27,28}$ Briefly, treated cells were washed with PBS and incubated in medium containing WST-1 at the working concentration as instructed in the user manuals. After 2 hours, the change in optical absorbance was recorded in a microplate reader at $450 \mathrm{~nm}$ (Sunrise Absorbance Reader, Tecan, Männedorf, Switzerland), with $650 \mathrm{~nm}$ as the reference wavelength. The half maximal inhibitory concentration $\left(\mathrm{IC}_{50}\right)$ was calculated mathematically by extrapolation. ${ }^{18}$

\section{Bright-field microscopy}

To assess cell morphology, cells were seeded on number 1 , $13 \mathrm{~mm}$ diameter glass coverslips (catalog number G402, ProSciTech Proprietary Limited, Kirwan, QLD, Australia) and allowed to attach for 24 hours. Cells were then treated with Fe@ $\mathrm{Au}$ for 24 hours. OECM1 cells were treated with $10 \mu \mathrm{g} / \mathrm{mL}$ Fe@Au, while CRC cells were treated with $50 \mu \mathrm{g} / \mathrm{mL}$, which are about the half-maximal inhibitory doses. Cells were washed and fixed in 4\% paraformaldehyde in PBS for 15 minutes. Cells were then mounted on the glass slides and sealed with nail polish for imaging with differential interference contrast (DIC) microscopy (FV-1000, Olympus Corporation, Tokyo, Japan).

\section{Scanning electron microscopy}

Cells were grown on $13 \mathrm{~mm}$ Thermanox ${ }^{\mathrm{TM}}$ coverslips (catalog number 26028, Thermanox, Nunc, Rochester, NY, USA) and then treated for 24 hours with a corresponding concentration of Fe@Au (ie, $10 \mu \mathrm{g} / \mathrm{mL}$ Fe@Au for OECM1 or $50 \mu \mathrm{g} / \mathrm{mL}$ Fe@Au for CRC). Cells were then fixed with 2\% glutaraldehyde (catalog number G5882, Sigma-Aldrich) for 1 hour at $4^{\circ} \mathrm{C}$. Samples were washed twice in PBS, followed by secondary fixation in 1\% osmium tetroxide in PBS for 1 hour at $4^{\circ} \mathrm{C}$, in the absence of light. Subsequently, samples were dehydrated in graded ethanol solutions. Cells were soaked in $30 \%, 50 \%, 70 \%, 80 \%, 90 \%$, and $95 \%$ ethanol for 5 minutes each and then $100 \%$ ultrapure ethanol for 5 minutes; with the soak in $100 \%$ ultrapure ethanol repeated twice more. The specimens were then immersed for 3 minutes in $100 \%$ hexamethyldisilazane (HMDS) (catalog number H4875, Sigma-Aldrich). Dried samples were then coated with $5 \mathrm{~nm}$ of platinum by using a sputter coater (EmiTech K550X; GaLa Instrument, Bad Schwalbach, Germany).

\section{Apoptosis and necrosis assay}

The apoptotic and necrotic cells were analyzed with the Dead Cell Apoptosis Kit (catalog number V13245, Invitrogen), following the manufacturer's instructions. ${ }^{29}$ Cells treated with $10 \mathrm{ng} / \mathrm{mL}$ tumor necrosis factor (catalog number 210TA, R\&D Systems, Minneapolis, MN, USA) and $1 \mu \mathrm{g} / \mathrm{mL}$ of cycloheximide (catalog number 01810 , Fluka Chemie, GmbH, Deisenhofen, Germany) for 4 hours were used as the positive control for apoptosis. Briefly, the treated cells were harvested in cold PBS and stained with propidium iodide (PI) and Alexa Fluor ${ }^{\circledR} 488$ annexin V. After 15 minutes, the stained cells were analyzed by flow cytometry (FACScan, BD Biosciences, Franklin Lakes, NJ, USA), measuring the fluorescence emission at $530 \mathrm{~nm}$ and $575 \mathrm{~nm}$ under $488 \mathrm{~nm}$ excitation.

\section{Quantification of Fe@Au internalization via inductively coupled plasma optical emission spectrometry}

The 500,000 OECM1 and CRC cells were seeded on plastic culture dishes (catalog number 5550300, Milian USA, Gahanna, OH, USA) and allowed to adhere for 24 hours. Fe@Au nanoparticles were added to the culture medium at a final concentration of $50 \mu \mathrm{g} / \mathrm{mL}$. The cells were then washed, counted, and dissolved in a mixture of $70 \%$ nitric acid (catalog number 84382, Sigma-Aldrich) and 36\% hydrochloric acid (catalog number H7020, Sigma-Aldrich) in a volume ratio of $2: 3$. Samples were digested at $60^{\circ} \mathrm{C}$ for 4 hours. Later, the digested samples were analyzed for iron and gold content by using inductively coupled plasma optical emission spectrometry (ICP-OES) (OPTIMA 7300; PerkinElmer, Waltham, MA, USA).

\section{Flow cytometry}

To assess cell-cycle progression, cells were harvested by using $0.25 \%$ trypsin with $1 \mathrm{mM}$ of ethylene diamine tetraacetic acid (EDTA) (catalog number 25200-256, Invitrogen) and fixed in $70 \%$ ice-cold ethanol overnight. The fixed cells were then centrifuged to thoroughly remove the ethanol. Subsequently, the cells were washed twice in $5 \mathrm{~mL}$ PBS and 
resuspended in $1 \mathrm{~mL}$ of PI-staining solution for 15 minutes at $37^{\circ} \mathrm{C}$. The staining solution comprised $20 \mu \mathrm{g} / \mathrm{mL}$ PI (catalog number 81845, Sigma-Aldrich), 0.1\% Triton X-100 (catalog number T8787, Sigma-Aldrich), and $0.2 \mathrm{mg} / \mathrm{mL}$ of RNase A (catalog number R4642, Sigma-Aldrich) in PBS. Samples were subsequently analyzed by using flow cytometry (FACScan, BD Biosciences).

For flow cytometry studies to assess mitochondrial membrane potential, JC-1 (5,5,6,60-tetrachloro-1,10,3,30tetraethylbenzimidazolylcarbocyanine) was used to estimate the polarization status of mitochondria. The aggregated form of JC-1 has maximal absorption and emission at $585 \mathrm{~nm}$ and $590 \mathrm{~nm}$, respectively, while the monomer occurs at $510 \mathrm{~nm}$ and $527 \mathrm{~nm}$. Staining was performed by using a mitochondria-staining kit (catalog number CS0390, SigmaAldrich), according to standard instructions. Briefly, cells were treated with their specific $\mathrm{IC}_{50}$ drug concentration for an assigned period of time. OECM1 cells were treated with $10 \mu \mathrm{g} / \mathrm{mL}$ of $\mathrm{Fe} @ \mathrm{Au}$, and CRC cells were treated with $50 \mu \mathrm{g} / \mathrm{mL}$ of $\mathrm{Fe} @ \mathrm{Au}$. Later, cells were harvested, stained with the kit, and analyzed by flow cytometry at the $530 \mathrm{~nm}$ and $590 \mathrm{~nm}$ emission wavelengths.

\section{Statistical analysis}

Data are presented as means or means \pm standard error (SE). Statistical differences were evaluated using analysis of variance and Student's $t$-test. The $P$-values are provided in context when above 0.0001 . All data were obtained from at least three independent experiments.

\section{Results}

\section{Differential cytotoxicity of Fe@Au in CRC cells}

The Fe@Au nanoparticles used in this study have been carefully characterized and reported in our previous studies, ${ }^{17,18}$ during which we found that CRC cells were less sensitive to $\mathrm{Fe} @ \mathrm{Au}$ treatment than oral cancer cells. ${ }^{17}$ Therefore, the oral cancer cell line OECM1 was used in this study as a positive control for the in-depth assessment of the cancer-specific cytotoxicity of Fe@Au across the different cancer cell lines investigated. Caco-2, HT-29, and SW480 colorectal adenocarcinoma cells were used in this study to investigate the different response of CRC versus oral cancer to Fe@Au treatment for 48 hours. As shown in Figure 1, the $\mathrm{IC}_{50}$ of OECM1, Caco-2, HT-29, and SW480 cells is $3.5,42.9,80.5$, and $39.0 \mu \mathrm{g} / \mathrm{mL}$, respectively. Although the nanoparticles caused a dose-dependent cytotoxic response both in the CRC and OECM1 cells after 48 hours, the $\mathrm{CRC}$ cells were at least an order of magnitude less sensitive,

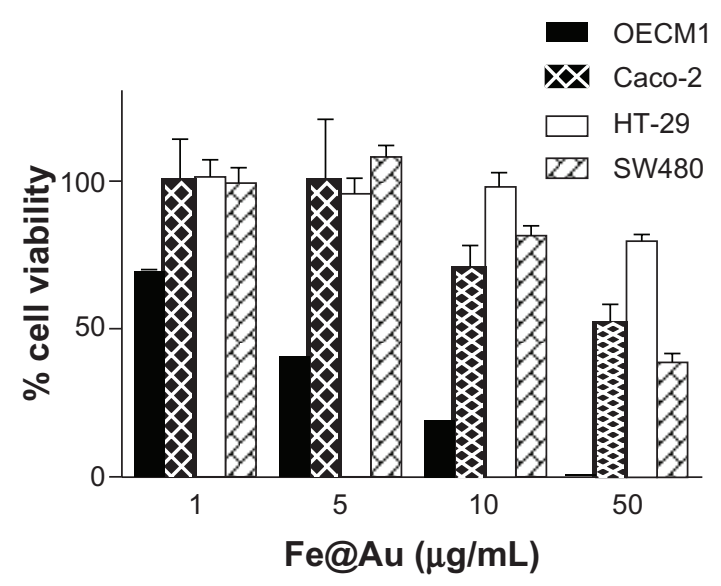

Figure I Bar graph illustrating the different cytotoxicity of Fe@Au nanoparticles in CRC and OECMI cells, as assessed with the WST-I assay.

Notes: The CRC cell lines Caco-2, HT-29, and SW480 are directly compared with the OECMI oral cancer cell line. From this graph, it becomes clear that CRC cells are less sensitive to Fe@Au treatment (Caco-2 cells, $P=0.0253$; HT-29, $P=0.0376$; SW480, $P=0.060 \mathrm{I}$; $t$-test), compared to OECMI cells.

Abbreviations: CRC, colorectal cancer; Fe, iron; Au, gold; WST-I, water-soluble tetrazolium salt.

based on $\mathrm{IC}_{50}$ dosage, to Fe $@$ Au treatment than OECM1 cells (Caco-2, $P=0.0253$; HT-29, $P=0.0376$; SW480, $P=0.0601$; compared to OECM1, $t$-test).

In the subsequent experiments, we applied doses of $10 \mu \mathrm{g} /$ $\mathrm{mL}$ and $50 \mu \mathrm{g} / \mathrm{mL}$ of $\mathrm{Fe} @ \mathrm{Au}$ to treat OECM1 and CRC cells respectively; these concentrations are close to the half-maximal inhibitory doses for the cell lines. Using a single dose of Fe@ $\mathrm{Au}$ in different $\mathrm{CRC}$ cells facilitates the scientific comparison between different cell lines and different experiments.

\section{Cell death and cell cycle analysis in Fe@Au treated cells}

Under bright-field microscopy (Figure 2) and high-resolution SEM(Figure 3), no structural difference in cell morphology was found when cells were treated for 24 hours - the time window to observe apoptosis and necrosis morphologically ${ }^{30,31}$ - and this was comparable to control conditions. The density of cells markedly decreased upon Fe@Au exposure, which is in accordance with the results shown in Figure 1. However, the molecular analysis of cells for apoptosis and necrosis showed that there was no statistically significant increase in the rate of apoptosis or necrosis in Fe@Au-treated cells (Figure 4). The slight, nonsignificant increase in apoptotic and necrotic cells clearly cannot account for the dramatic decrease in overall cell viability observed in Figure 1.

As the Fe@Au treatment diminished cell viability without inducing apoptotic or necrotic cell death, the inhibition of cell growth must therefore be considered. In our previous work, we demonstrated that $\mathrm{Fe} @$ Au retarded cell growth at the S-phase 


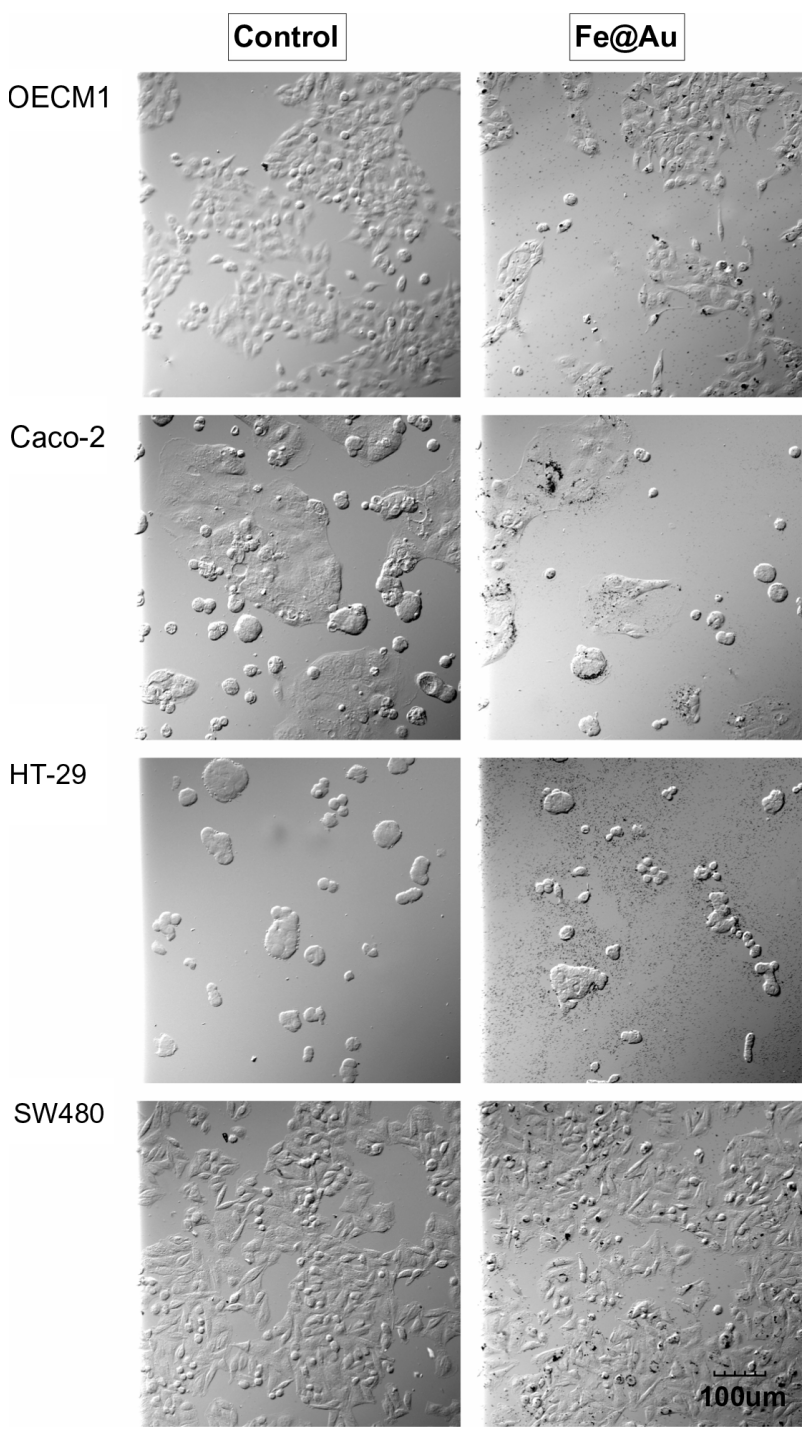

Figure 2 The bright-field optical images show the morphology of Fe@Autreated cells.

Notes: Except for a different cell density, the CRC and OECMI cells show no significant alteration in overall morphology after 24 hours of Fe@Au exposure. All images were recorded at the same magnification. Scale bar, $100 \mu \mathrm{m}$.

Abbreviations: $\mathrm{CRC}$, colorectal cancer; Fe, iron; Au, gold.

rather than causing cell death in OECM1 cells after a 24-hour treatment. ${ }^{17}$ Therefore, we next analyzed the cell cycle profile of Fe@Au-treated CRC. In Figure 5, OECM1 cells show S-phase delay, as found in our previous report. ${ }^{17}$ Intriguingly, $\mathrm{Fe} @$ Au caused a G1/G0 delay in CRC cells. Therefore, the decreased cell survival rate of Fe@Au-treated CRC cells is the result of growth inhibition, rather than cell death, which is consistent with our observations in oral cancer cells. ${ }^{17}$

\section{Fe@Au treatment and assessment of mitochondrial membrane potential}

We previously demonstrated that oral cancer cells, including OECM1, exposed to Fe@Au suffered a loss of mitochondria
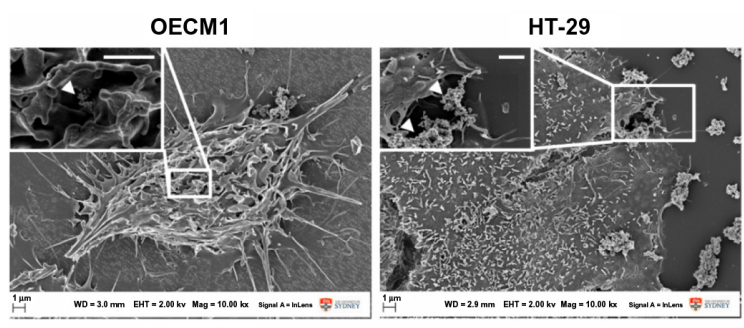

Caco-2
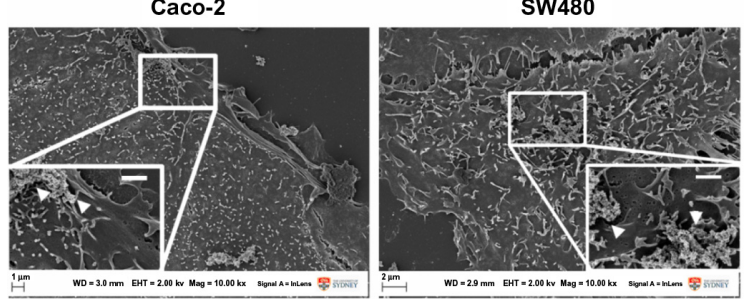

Figure 3 Fe@Au-treated cells visualized under intermediate- and high-magnification scanning electron microscopy.

Notes: At both magnifications, no significant fine-structure differences could be observed in the different cancer cells when treated with Fe@Au for 24 hours; ie, all cells remained well attached to the substratum and maintained their typical surface morphology, such as microvilli and numerous lamellipodia, corresponding to control conditions (data not shown). Insets illustrate the presence of discrete Fe@ Au clumps (white arrows) on the surface of the cells. Scale bars inset, I $\mu \mathrm{m}$. Abbreviations: Fe, iron; Au, gold.

membrane potential in 4 hours and 24 hours $^{18}$ and, therefore, induced cytotoxicity via mitochondria-mediated autophagy. ${ }^{17}$ To investigate whether Fe@Au nanoparticles cause cytotoxicity in CRC cells via a common mechanism, we used flow cytometry to analyze the status of mitochondria in Fe@Autreated CRC cells. Our data (Figure 6), however, revealed that Caco- 2 cells showed only a minor membrane potential loss after 24-hour treatment, while HT-29 and SW480 cells displayed no loss of membrane potential at all. To further explore the underlying mechanism of the Fe@Au-induced cytotoxicity in CRC, cells were treated with the mitochondria membrane potential transition blocker, CsA, and autophagy inhibitor, 3-MA. As shown in Figure 7, OECM1 and Caco-2 cells showed a similar response to the nanoparticle treatment. The 3-MA provided significant protection to the OECM1 and Caco- 2 cells from the cytotoxicity of Fe@Au, but not to the HT-29 and SW480 cells. However, CsA was found to restore the cytotoxicity of Fe@Au in OECM1 but not in CRC cell lines, in accordance with the JC-1 staining results. This observation suggests that $\mathrm{Fe} @$ Au-induced cytotoxicity in HT-29 and SW480 cells occurs via an alternative pathway compared with oral cancer cells.

\section{Fe@Au resistance in CRC cells versus oral cancer cells}

Next, we explored the amounts of Fe@Au internalized in CRC and OECM1 cells. Fe@Au-treated cells were 

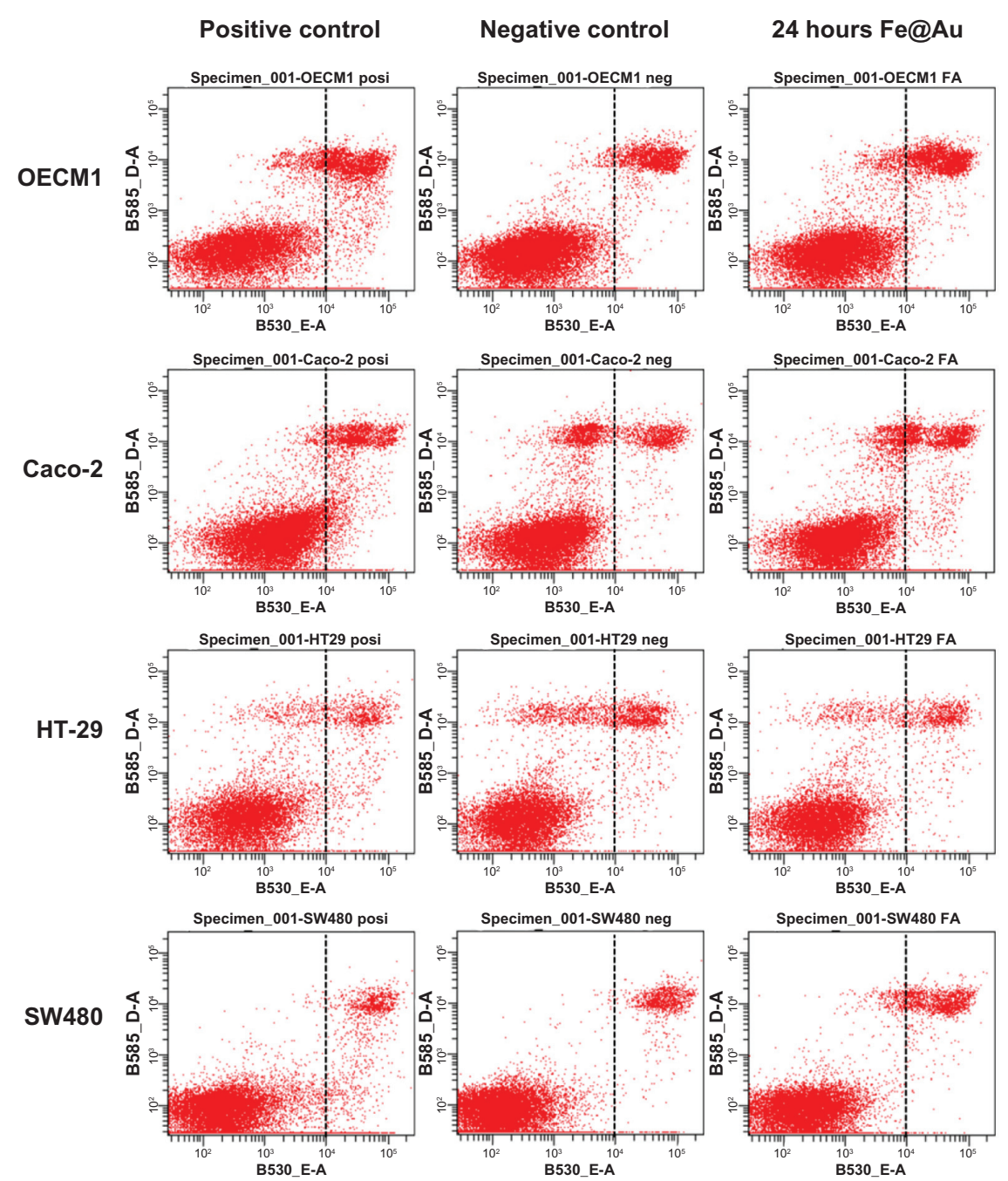

Figure 4 The apoptosis and necrosis of Fe@Au-treated cells were analyzed by flow cytometry.

Notes: The treated CRC cells show only a small increase in the number of apoptotic and necrotic cells. $10 \mu \mathrm{g} / \mathrm{mL}$ Fe@Au was used in OECMI; $50 \mu \mathrm{g} / \mathrm{mL}$ Fe@Au was used in CRC. Cells were treated for 24 hours.

Abbreviations: CRC, colorectal cancer; Fe, iron; Au, gold.

collected after specific exposure periods of up to 2 hours (internalization of most nanomaterials has been reported to plateau within 2 hours $^{32,33}$ ), and then cells were lysed and analyzed by ICP-OES.

In Figure 8, at 2 hours posttreatment, Caco- 2 cells show the highest amount of internalized $\mathrm{Au}$ and Fe, followed by SW480, OECM1, and then HT-29 cells. The highest internalization rate within the first hour occurred in SW480, followed by OECM1, HT-29, and then Caco-2 cells. It is noteworthy that neither the amount nor the rate of $\mathrm{Au}$ and $\mathrm{Fe}$ internalization explains the differential responses between OECM1 and CRC cell lines, as depicted in Figure 1. Intriguingly, we found that the internalization profiles of $\mathrm{Au}$ and Fe were different between OECM1 and HT-29. In accordance with our previous research, ${ }^{17,18}$ the nanoparticles served as a package of $\mathrm{Fe}$ and $\mathrm{Au}$. The ratio of internalized $\mathrm{Au}$ and $\mathrm{Fe}$ elements should be constant, on average, and if the cells absorb a large number of unchanged nanoparticles. Intriguingly, when plotting the molar ratio of $\mathrm{Fe}$ to Au at different time points (Figure 9), it becomes apparent that different ratios occur between the different cell lines.

In particular, the Fe to Au ratio of treated HT- 29 cells is significantly lower than the ratio of treated OECM1 cells at all-time points investigated $(P=0.0210 ; t$-test). This suggests that the proportion of intracellular $\mathrm{Fe}$ and Au plays an important role in the relative sensitivity versus the resistance of cells to Fe@Au treatment.

Based on these findings, we propose that the ability of cells to deal with Fe may be the main reason for the different cytotoxic response observed in the cell lines studied. To test this hypothesis, cells were treated with Fe-only nanoparticles. 

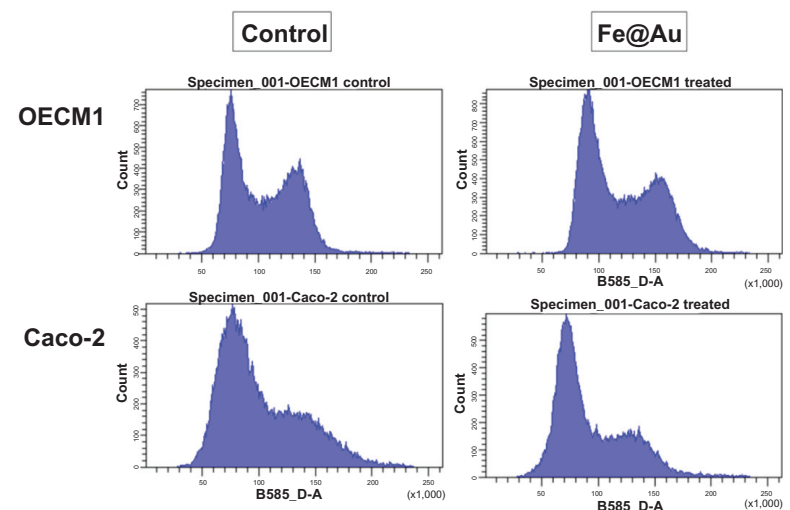

HT-29
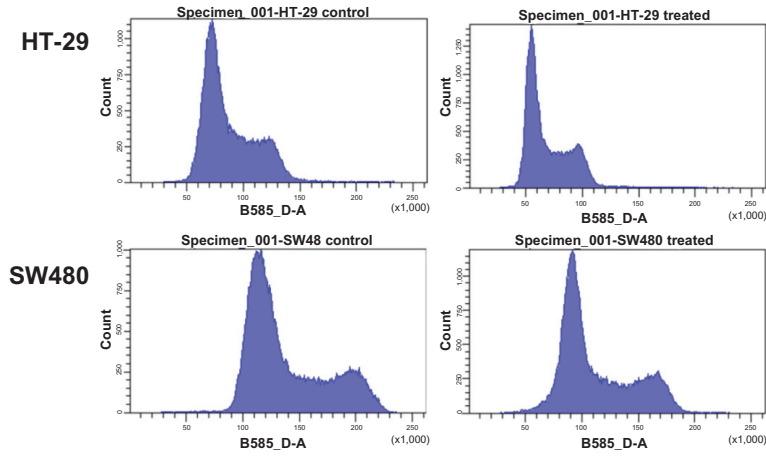

Figure 5 The cell cycle of Fe@Au treated cells analyzed via flow cytometry. Notes: When compared to the S-phase delay of OECMI cells, Fe@Au-treated CRC cells show a sharper peak at the left after 24 hours of treatment, indicating cell-cycle retardation at the GI/G0 phase.

Abbreviations: CRC, colorectal cancer; Fe, iron; Au, gold.
The cytotoxic effect was directly compared with Fe@Au. Figure 10 provides support for this hypothesis, revealing that OECM1 and Caco-2 cells are more sensitive to Fe-only nanoparticles at most concentrations tested. However, the HT-29 cells show a significant resistance to Fe-only nanoparticles $(P=0.0205$; $t$-test). SW480 cells are more resistant to Fe-only nanoparticles than to Fe@Au, only at the concentration of $50 \mu \mathrm{g} / \mathrm{mL}(P=0.0041$; $t$-test $)$. This observation supports the idea that the resistance to Fe-only nanoparticles is highly correlated to the relative sensitivity or resistance of the different cells to the Fe@Au nanoparticles. We also noticed that the presence of $\mathrm{Au}$ in $\mathrm{Fe} @$ Au nanoparticles seems to play an important role in the Fe@Au-induced cytotoxicity in the Fe-resistant HT-29 and SW480 cells.

\section{Discussion}

In this study, we investigated the mechanisms of CRC cell resistance to treatments with $\mathrm{Fe} @ \mathrm{Au}$ nanoparticles and directly compared the responses with the Fe@Au-sensitive OECM1 oral cancer cell line. We found that Fe@Au retards growth in both CRC and OECM1 cell lines, but the Fe@Autreated CRC cells are arrested in G1/G0 phase, rather than in the S-phase, as observed in the treated OECM1 cell line. Furthermore, we observed that the mitochondrial responses

\section{Negative control}

Positive control

4 hours Fe@Au

24 hours Fe@Au

Caco-2
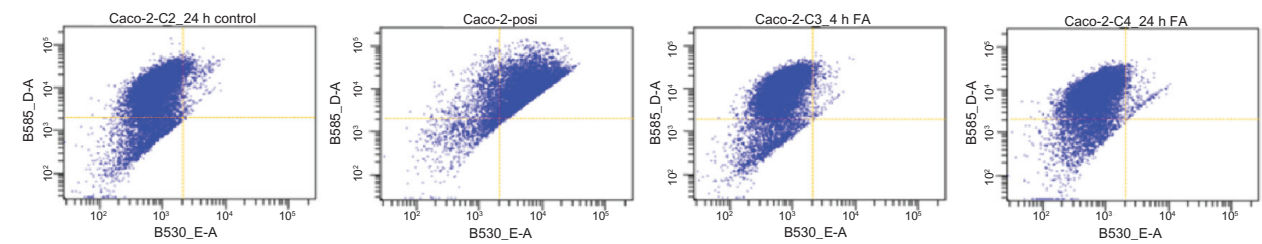

HT-29
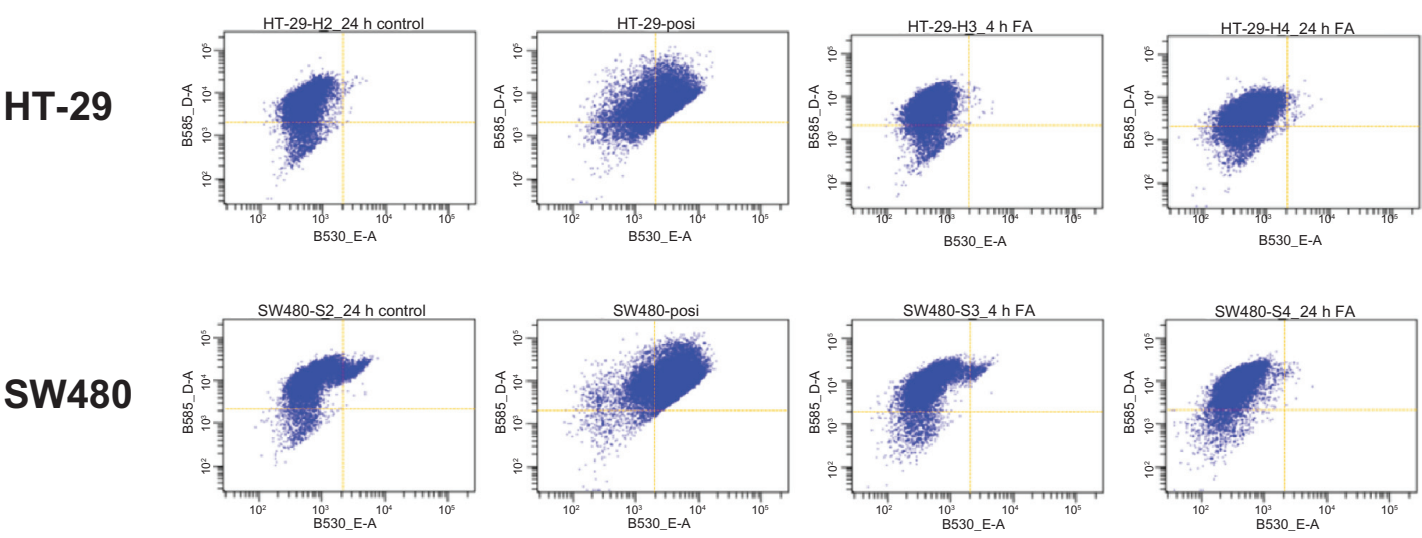

Figure 6 The mitochondrial response examined before and after Fe@Au treatment.

Notes: Among the CRC cell lines investigated, only Caco-2 cells show a minor increase in mitochondrial membrane potential after 24 hours of treatment. '4 hours Fe@Au' indicates a $50 \mu \mathrm{g} / \mathrm{mL}$ Fe@Au treatment for 4 hours, and '24 hours Fe@Au' indicates a $50 \mu \mathrm{g} / \mathrm{mL}$ Fe@Au treatment for 24 hours.

Abbreviations: Fe, iron; Au, gold; CRC, colorectal cancer. 


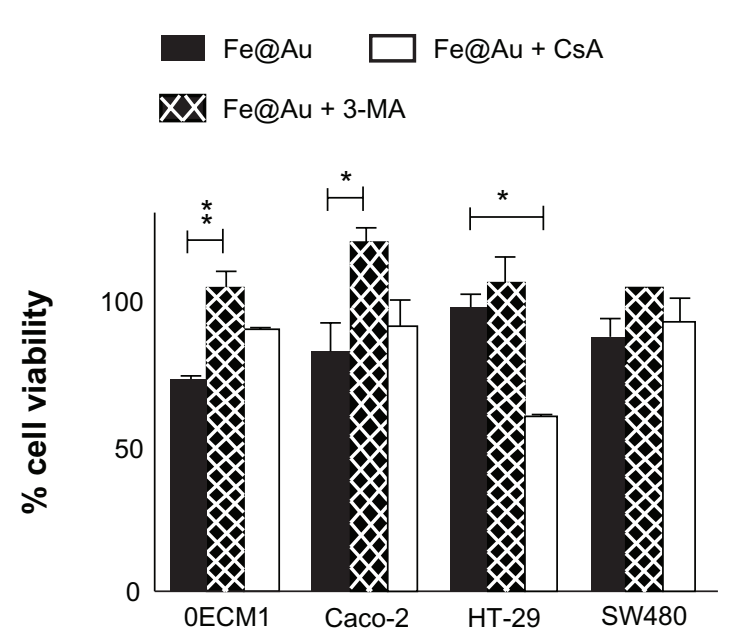

Figure 7 Bar graph illustrating the overall viability of Fe@Au-treated cancer cells, and the influence of the mitochondrial membrane potential blocker CsA and the autophagy inhibitor 3-MA.

Notes: WST-I cell viability analysis was performed after 48 hours with the assigned treatment; ie, either in the presence with $5 \mu \mathrm{M}$ CsA or $10 \mathrm{mM} 3-\mathrm{MA}$. The cell viability of Fe@Au + CsA and Fe@Au + 3-MA was calculated according to the matching control conditions of CsA alone and 3-MA alone. ${ }^{*} P<0.05$; $* * P<0.01$; $t$-test.

Abbreviations: Fe, iron; Au, gold; CsA, cyclosporin A; 3-MA, 3-methyladenine; WST-I, water-soluble tetrazolium salt.

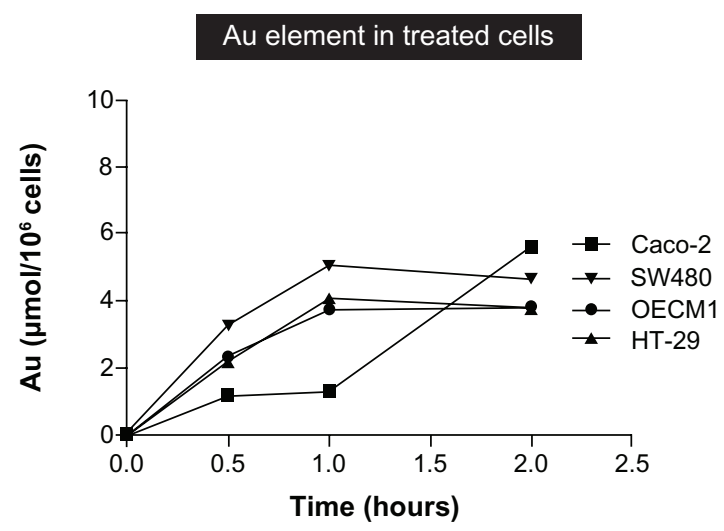

Fe element in treated cells

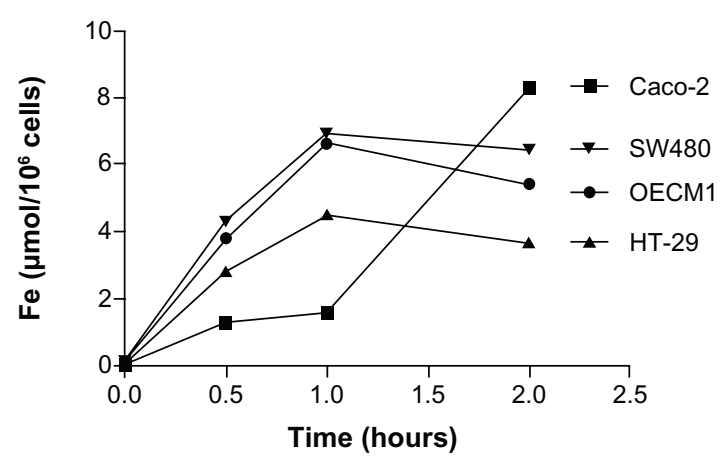

Figure 8 Line graph summarizes the internalization profiles of Fe@Au nanoparticles as assessed by ICP-OES.

Notes: Cells were treated with $50 \mu \mathrm{g} / \mathrm{mL}$ of Fe@Au for the assigned times; the cells were harvested; and the Fe and Au concentration was analyzed. From this graph, it becomes clear that the total amount of internalized Fe or Au is not proportional to the cytotoxicity observed in individual cells.

Abbreviations: ICP-OES, inductively coupled plasma optical emission spectrometry; Fe, iron; Au, gold.

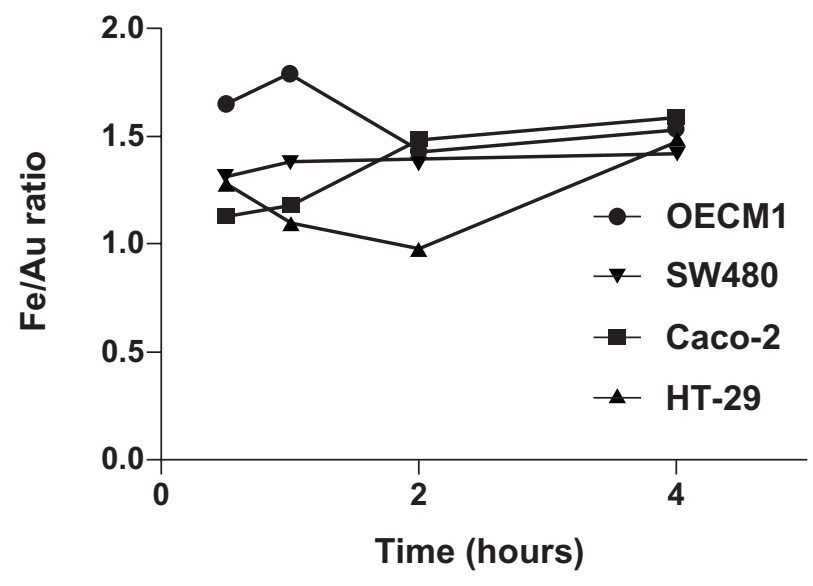

Figure 9 The ratio of internalized $\mathrm{Fe}$ and Au elements was explored at different times. Notes: The molar ratio of $\mathrm{Fe}$ to $\mathrm{Au}$ is used as an index of internalized elements. In the early time points ( 30 minutes and I hour), the OECMI cells show the highest Fe:Au ratio. Interestingly, the Fe:Au ratio in HT-29 cells is significantly lower than in OECMI cells $(P=0.0210$; $t$-test), which correlates with HT-29 cells' extreme resistance to Fe@Au treatment (Figure I).

Abbreviations: Fe, iron; Au, gold.

to the Fe@Au treatment were distinct in different CRC cells, with only Fe@Au-treated Caco-2 cells showing any mitochondrial membrane potential loss within 24 hours (Figure 6). Furthermore, the mitochondria membrane potential blocker, CsA, could not protect the HT-29 and SW480 cells from the damage caused by Fe@Au; while 3-MA significantly restored the Fe@Au induced cytotoxicity in Caco-2 cells $(P<0.05)$. According to the difference in uptake profiles of $\mathrm{Fe}$ and $\mathrm{Au}$ (Figure 9), as well as to the responses to Fe-only nanoparticle treatment (Figure 10), Caco-2 cells were found to be more sensitive to $\mathrm{Fe}$ alone compared to the other two CRC cell lines. Hence, Fe@Au nanoparticles apparently induce cytotoxicity in HT-29 and SW480 cells through pathways different to the ones observed in OECM1 cells and to Caco-2 cells. Given these results and the previously demonstrated importance of $\mathrm{Fe}$ in the cancer-preferential cytotoxicity ${ }^{17}$ that occurs in Fe@Au-sensitive cells, it seems evident that the cells better able to deal with Fe will display greater resistance to Fe@Au treatment.

It has been reported previously that $\mathrm{Au}$ is highly biocompatible in the majority of biomedical research settings. ${ }^{23,34}$ Yet, in this study, we were able to demonstrate that Au plays another role besides acting as the passivation layer on the Fecore nanoparticles, as Au was found essential for the toxicity of Fe@Au in cell lines, such as HT-29 and SW480, which are resistant to Fe-only treatments. It is interesting to note that the work concerning the cytotoxicity and/or biocompatibility of Au was mostly performed in cancer cell lines. ${ }^{35}$

There are two possible explanations: (1) the Au keeps a higher level of nonoxidized Fe in the Fe@Au nanoparticles, 

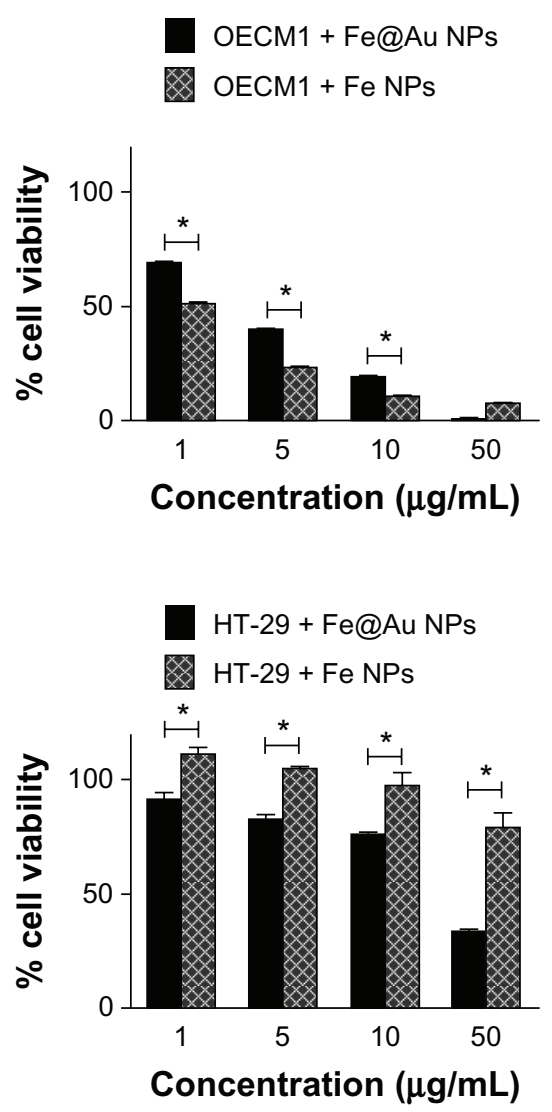
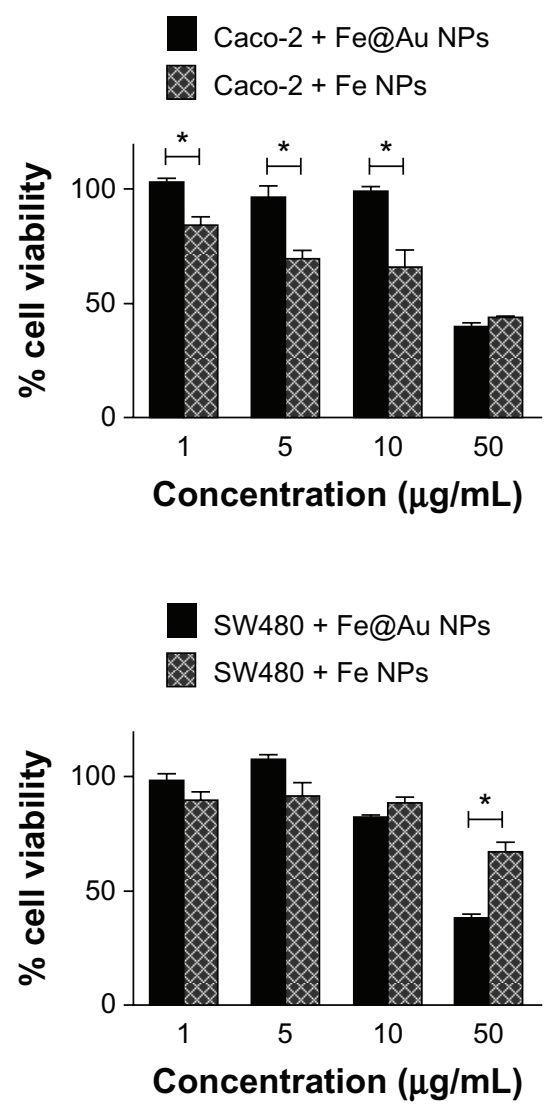

Figure 10 Cytotoxic response of different cell lines shows different sensitivity toward Fe@Au or Fe-only nanoparticle treatments.

Notes: CRC and OECMI were treated with Fe-only nanoparticles (Fe NPs) and Fe@Au nanoparticles (Fe@Au NPs) for 48 hours, analyzed by WST-I. OECMI and Caco-2 are more sensitive to Fe nanoparticles; whereas, the other CRC, (HT-29 and SW480) are more resistant to Fe nanoparticles than to Fe@Au nanoparticles. ( $* P<0.05$; $t$-test).

Abbreviations: Fe, iron; Au, gold; NPs, nanoparticles; WST-I, water-soluble tetrazolium salt; CRC, colorectal cancer.

leading to higher redox activity within the cells than the Feonly NPs, and that this accounts for the preferential effects of the coated NPs; and/or (2) there is some synergistic effect of the $\mathrm{Au}$ and Fe elements and/or ions within the cells.

Our research also suggests that the resistance to $\mathrm{Fe} @ \mathrm{Au}$ treatment may rely on the ability to cope with Fe. Fe-resistant cells may either have a more robust mitochondrial system or a more effective efflux system, such as ferroportin, to eliminate the Fe. Ferroportin is a protein that serves as a transmembrane ion channel to enable Fe efflux from cells ${ }^{36}$ and is expressed in hepatic cells, macrophages, and also in enterocytes. ${ }^{37}$ More research is needed to further investigate this hypothesis and elucidate the full mechanisms of $\mathrm{Fe} @ \mathrm{Au}$ sensitivity in cancer cells.

\section{Conclusion}

In our previous study, nonoxidized Fe in $\mathrm{Fe} @ \mathrm{Au}$ nanoparticles was found to play an essential role in the cancerpreferential cytotoxicity of Fe@Au in oral cancer models, in vitro and in vivo. Here, Fe@Au nanoparticles have demonstrated a similar, but less intensive, cytotoxicity in CRC cells. We found that the cytotoxicity of the nanoparticles was lower in particle CRC cells that were able to reduce their internal levels of Fe. Furthermore, the Au coatings significantly enhanced the cytotoxicity of nanoparticles in selected CRC cells. Therefore, both the Fe core and Au shell of $\mathrm{Fe} @ \mathrm{Au}$ are critical for the anticancer property observed in cancer cells.

\section{Acknowledgments}

DBS is indebted to Taiwan National Science Council (grant number 101-2120-M-006-008-, 101-2314-B-006-048-MY3) and the Taiwan Department of Health (grant number DOH101TD-PB-111-NSC004, DOH102-TD-PB-111-TM017). PCW thanks Taiwan National Science Council for grant support (101-2320-B-006-004). We also thank the Australian Research Council for a Discovery Project Grant (DP0985059) to PT and FB, as well as the Cancer Institute NSW (08/RFG/1-29) for supporting our work. We acknowledge the facilities as well as scientific and technical assistance from staff at the Australian Microscopy and Microanalysis Research Facility at the Australian Centre for Microscopy and Microanalysis, the 
University of Sydney. All animal studies and human primary cell cultures were performed in accordance with the University of Cheng-Kung Internal Review Board for Ethics and Human Research (ethical approval No 97218).

\section{Disclosure}

The authors report no conflicts of interest in this work.

\section{References}

1. Singh N, Jenkins GJ, Asadi R, Doak SH. Potential toxicity of superparamagnetic iron oxide nanoparticles (SPION). Nano Rev. 2010;1:5358.

2. Gupta AK, Gupta M. Synthesis and surface engineering of iron oxide nanoparticles for biomedical applications. Biomaterials. 2005;26(18): 3995-4021.

3. Chen B, Wu W, Wang X. Magnetic iron oxide nanoparticles for tumor-targeted therapy. Curr Cancer Drug Targets. 2011;11(2): 184-189.

4. Shieh DB, Cheng FY, Su CH, et al. Aqueous dispersions of magnetite nanoparticles with $\mathrm{NH} 3+$ surfaces for magnetic manipulations of biomolecules and MRI contrast agents. Biomaterials. 2005;26(34): 7183-7191.

5. Chou SW, Shau YH, Wu PC, Yang YS, Shieh DB, Chen CC. In vitro and in vivo studies of FePt nanoparticles for dual modal CT/MRI molecular imaging. J Am Chem Soc. 2010;132(38):13270-13278.

6. Wu PC, Wang WS, Huang YT, et al. Porous iron oxide based nanorods developed as delivery nanocapsules. Chemistry. 2007;13(14): 3878-3885.

7. Ito A, Honda H, Kobayashi T. Cancer immunotherapy based on intracellular hyperthermia using magnetite nanoparticles: a novel concept of "heat-controlled necrosis" with heat shock protein expression. Cancer Immunol Immunother. 2006;55(3):320-328.

8. Jain TK, Reddy MK, Morales MA, Leslie-Pelecky DL, Labhasetwar V. Biodistribution, clearance, and biocompatibility of iron oxide magnetic nanoparticles in rats. Mol Pharm. 2008;5(2):316-327.

9. Karlsson HL, Cronholm P, Gustafsson J, Möller L. Copper oxide nanoparticles are highly toxic: a comparison between metal oxide nanoparticles and carbon nanotubes. Chem Res Toxicol. 2008;21(9): $1726-1732$.

10. Reimer P, Balzer T. Ferucarbotran (Resovist): a new clinically approved RES-specific contrast agent for contrast-enhanced MRI of the liver: properties, clinical development, and applications. Eur Radiol. 2003;13(6):1266-1276.

11. Carpenter EE, Sangregorio C, O'Connor CJ. Effects of shell thickness on blocking temperature of nanocomposites of metal particles with gold shells. IEEE Trans Magn. 1999;35(5):3496-3498.

12. Carpenter EE, Sims JA, O'Connor CJ. Sequential synthesis of gold coated iron nanoparticles using reverse micelles. Abstr Paper Am Chem Soc Natl Meet. 1999;218:U441.

13. Cho SJ, Jarrett BR, Louie AY, Kauzlarich SM. Gold-coated iron nanoparticles: a novel magnetic resonance agent for $\mathrm{T}_{1}$ and $\mathrm{T}_{2}$ weighted imaging. Nanotechnology. 2006;17(3):640-644.

14. Kayal S, Ramanujan RV. Anti-cancer drug loaded iron-gold coreshell nanoparticles (Fe@Au) for magnetic drug targeting. J Nanosci Nanotechnol. 2010;10(9):5527-5539.

15. Park HY, Schadt MJ, Wang L, et al. Fabrication of magnetic core@ shell Fe oxide@Au nanoparticles for interfacial bioactivity and bioseparation. Langmuir. 2007;23(17):9050-9056.

16. Kim DH, Rozhkova EA, Rajh T, Bader SD, Novosad V. Synthesis of hybrid gold/iron oxide nanoparticles in block copolymer micelles for imaging, drug delivery, and magnetic hyperthermia. IEEE Transactions, Magnetics. 2009;45(10):4821-4824.
17. Wu YN, Chen DH, Shi XY, et al. Cancer-cell-specific cytotoxicity of non-oxidized iron elements in iron core-gold shell NPs. Nanomedicine. 2011;7(4):420-427.

18. Wu YN, Yang LX, Shi XY, et al. The selective growth inhibition of oral cancer by iron core-gold shell nanoparticles through mitochondriamediated autophagy. Biomaterials. 2011;32(20):4565-4573.

19. Thurber A, Wingett DG, Rasmussen JW, et al. Improving the selective cancer killing ability of $\mathrm{ZnO}$ nanoparticles using $\mathrm{Fe}$ doping. Nanotoxicology. 2012;6(4)440-452.

20. Lin J, Zhou W, Kumbhar A, et al. Gold-coated iron (Fe@Au) nanoparticles: synthesis, characterization, and magnetic field-induced self-assembly. J Solid State Chem. 2001;159(1):26-31.

21. Ravel B, Carpenter EE, Harris VG. Oxidation of iron in iron/gold core/ shell nanoparticles. J Appl Phys. 2002;91(10):8195-8197.

22. Zhou WL, Carpenter EE, Lin J, Kumbhar A, Sims J, O'Connor CJ. Nanostructures of gold coated iron core-shell nanoparticles and the nanobands assembled under magnetic field. Eur Phys J D. 2001;16(1): 289-292.

23. Tshikhudo TR, Wang Z, Brust M. Biocompatible gold nanoparticles. Materials Science and Technology. 2004;20(8):980-984.

24. Li F, Vipulanandan C, Mohanty KK. Microemulsion and solution approaches to nanoparticle iron production for degradation of trichloroethylene. Colloids and Surfaces A: Physicochemical and Engineering Aspects. 2003;223(1-3):103-112.

25. Wu L, Shamsuzzoha M, Ritchie SMC. Preparation of cellulose acetate supported zero-valent iron nanoparticles for the dechlorination of trichloroethylene in water. J Nanopart Res. 2005;7(4-5):469-476.

26. Peng YC, Kuo HS, Tsai HD, Yang YP, Lin YL. The lethal effect of bis-type azridinylnaphthoquinone derivative on oral cancer cells (OEC-M1) associated with anti-apoptotic protein bcl-2. Bioorg Med Chem. 2006;14(1):263-272.

27. Wörle-Knirsch JM, Pulskamp K, Krug HF. Oops they did it again! Carbon nanotubes hoax scientists in viability assays. Nano Lett. 2006;6(6):1261-1268.

28. Schröterová L, Králová V, Voráčová A, Hašková P, Rudolf E, Červinka M. Antiproliferative effects of selenium compounds in colon cancer cells: comparison of different cytotoxicity assays. Toxicol In Vitro. 2009;23(7):1406-1411.

29. Allen RT, Hunter WJ 3rd, Agrawal DK. Morphological and biochemical characterization and analysis of apoptosis. J Pharmacol Toxicol Methods. 1997;37(4):215-228.

30. Tang W, Zhang Y, Qian C, Yuan Z, Du J. Induction and mechanism of apoptosis by hydroxycamptothecin in human Tenon's capsule fibroblasts. Invest Ophthalmol Vis Sci. 2012;53(8):4874-4880.

31. Vangestel C, Peeters M, Mees G, et al. In vivo imaging of apoptosis in oncology: an update. Mol Imaging. 2011;10(5):340-358.

32. Jiang L, Li X, Liu L, Zhang Q. Cellular uptake mechanism and intracellular fate of hydrophobically modified pullulan nanoparticles. Int J Nanomedicine. 2013;8:1825-1834.

33. Summers HD, Holton MD, Rees P, Williams PM, Thornton CA. Analysis of quantum dot fluorescence stability in primary blood mononuclear cells. Cytometry A. 2010;77(10):933-939.

34. Chen M, Yamamuro S, Farrell D, Majetich SA. Gold-coated iron nanoparticles for biomedical applications. J Appl Phys. 2003;93(10): 7551-7553.

35. Uboldi C, Bonacchi D, Lorenzi G, et al. Gold nanoparticles induce cytotoxicity in the alveolar type-II cell lines A549 and NCIH441. Part Fibre Toxicol. 2009;6:18.

36. Vyoral D, Petrák J. Hepcidin: a direct link between iron metabolism and immunity. Int J Biochem Cell Biol. 2005;37(9):1768-1773.

37. Abboud S, Haile DJ. A novel mammalian iron-regulated protein involved in intracellular iron metabolism. J Biol Chem. 2000;275(26): 19906-19912. 
International Journal of Nanomedicine

Dovepress

\section{Publish your work in this journal}

The International Journal of Nanomedicine is an international, peerreviewed journal focusing on the application of nanotechnology in diagnostics, therapeutics, and drug delivery systems throughou the biomedical field. This journal is indexed on PubMed Central, MedLine, CAS, SciSearch ${ }^{\circledR}$, Current Contents ${ }^{\circledR} /$ Clinical Medicine,
Journal Citation Reports/Science Edition, EMBase, Scopus and the Elsevier Bibliographic databases. The manuscript management system is completely online and includes a very quick and fair peer-review system, which is all easy to use. Visit http://www.dovepress.com/ testimonials.php to read real quotes from published authors.

Submit your manuscript here: http://www.dovepress.com/international-journal-of-nanomedicine-journal 